\title{
The HLA-DQß1 insertion is a strong achalasia risk factor and displays a geospatial north-south gradient among Europeans
}

\author{
Jessica Becker 1,2,32, Stephan L Haas ${ }^{3,32}$, Anna Mokrowiecka ${ }^{4,32}$, Justyna Wasielica-Berger ${ }^{5}$, Zeeshan Ateeb ${ }^{3}$, \\ Jonna Bister ${ }^{3}$, Peter Elbe ${ }^{3}$, Marek Kowalski ${ }^{4}$, Magdalena Gawron-Kiszka ${ }^{6}$, Marek Majewski ${ }^{7}$, Agata Mulak ${ }^{8}$, \\ Maria Janiak $^{9}$, Mira M Wouters ${ }^{10}$, Till Schwämmle ${ }^{1,2}$, Timo Hess ${ }^{1,2}$, Lothar Veits $^{11}$, Stefan Niebisch ${ }^{12}$, \\ José L Santiago $^{13}$, Antonio Ruiz de León ${ }^{14}$, Julio Pérez de la Serna ${ }^{14}$, Elena Urcelay ${ }^{13}$, Vito Annese ${ }^{15}$, \\ Anna Latiano ${ }^{16}$, Uberto Fumagalli ${ }^{17}$, Riccardo Rosati ${ }^{18}$, Luigi Laghi ${ }^{19}$, Rosario Cuomo ${ }^{20}$, Frank Lenze ${ }^{21}$, \\ Giovanni Sarnelli ${ }^{20}$, Michaela Müller ${ }^{22}$, Burkhard HA von Rahden ${ }^{23}$, Cisca Wijmenga ${ }^{24}$, Hauke Lang ${ }^{25}$, \\ Kamila Czene $^{26}$, Per Hall ${ }^{26}$, Paul IW de Bakker ${ }^{27,28}$, Michael Vieth ${ }^{11}$, Markus M Nöthen ${ }^{1,2}$, \\ Henning G Schulz ${ }^{29}$, Krystian Adrych ${ }^{9}$, Anita Gąsiorowska ${ }^{4}$, Leszek Paradowski ${ }^{8}$, Grzegorz Wallner ${ }^{7}$, \\ Guy E Boeckxstaens ${ }^{10}$, Ines Gockel ${ }^{12}$, Marek Hartleb ${ }^{6}$, Srdjan Kostic ${ }^{3}$, Anna Dziurkowska-Marek ${ }^{6}$, \\ Mats Lindblad ${ }^{3}$, Magnus Nilsson ${ }^{3}$, Michael Knapp ${ }^{30}$, Anders Thorell ${ }^{31}$, Tomasz Marek ${ }^{6}$, Andrzej Dąbrowski ${ }^{5}$, \\ Ewa Małecka-Panas ${ }^{4,32}$ and Johannes Schumacher ${ }^{\star, 1,2,32}$
}

Idiopathic achalasia is a severe motility disorder of the esophagus and is characterized by a failure of the lower esophageal sphincter to relax due to a loss of neurons in the myenteric plexus. Most recently, we identified an eight-amino-acid insertion in the cytoplasmic tail of HLA-DQ $\beta 1$ as strong achalasia risk factor in a sample set from Central Europe, Italy and Spain. Here, we tested whether the HLA-DQ $\beta 1$ insertion also confers achalasia risk in the Polish and Swedish population. We could replicate the initial findings and the insertion shows strong achalasia association in both samples (Poland $P=1.84 \times 10^{-04}$, Sweden $P=7.44 \times 10^{-05}$ ). Combining all five European data sets - Central Europe, Italy, Spain, Poland and Sweden - the insertion is achalasia associated with $P_{\text {combined }}=1.67 \times 10^{-35}$. In addition, we observe that the frequency of the insertion shows a geospatial north-south gradient. The insertion is less common in northern (around 6-7\% in patients and $2 \%$ in controls from Sweden and Poland) compared with southern Europeans ( $16 \%$ in patients and $8 \%$ in controls from Italy) and shows a stronger attributable risk in the southern European population. Our study provides evidence that the prevalence of achalasia may differ between populations.

European Journal of Human Genetics (2016) 24, 1228-1231; doi:10.1038/ejhg.2015.262; published online 6 January 2016

\section{INTRODUCTION}

Idiopathic achalasia represents a motility disorder of the esophagus and is characterized by aperistalsis and a failure of the lower esophageal sphincter to relax due to a loss of neurons in the myenteric plexus. ${ }^{1}$ Although the cause of this neuronal degeneration is mainly unknown, autoimmune processes seem to be involved in individuals

${ }^{1}$ Institute of Human Genetics, University of Bonn, Bonn, Germany; ${ }^{2}$ Department of Genomics, Life \& Brain Center, University of Bonn, Bonn, Germany; ${ }^{3}$ Center for Digestive Diseases, Karolinska University Hospital, Stockholm, Sweden; ${ }^{4}$ Department of Digestive Tract Diseases, Medical University of Lodz, Lodz, Poland; ${ }^{5}$ Department of Gastroenterology and Internal Diseases, Medical University of Bialystok, Bialystok, Poland; ${ }^{6}$ Department of Gastroenterology and Hepatology, Medical University of Silesia, Katowice, Poland; ${ }^{7}$ Second Department of General Surgery, Medical University of Lublin, Lublin, Poland; ${ }^{8}$ Department of Gastroenterology and Hepatology, Wroclaw Medical University, Wroclaw, Poland; ${ }^{9}$ Department of Gastroenterology and Hepatology, Medical University of Gdansk, Gdansk, Poland; ${ }^{10}$ Translational Research Center for Gastrointestinal Disorders, Catholic University of Leuven, Leuven, Belgium; ${ }^{11}$ Institute of Pathology, Klinikum Bayreuth, Bayreuth, Germany; ${ }^{12}$ Department of Visceral, Transplant, Thoracic and Vascular Surgery, University Hospital of Leipzig, Leipzig, Germany; ${ }^{13}$ Department of Immunology, Instituto de Investigación Sanitaria del Hospital Clínico San Carlos (IdISSC), Madrid, Spain; ${ }^{14}$ Department of Gastroenterology, Instituto de Investigación Sanitaria del Hospital Clínico San Carlos (IdISSC), Madrid, Spain; ${ }^{15}$ Department of Gastroenterology, Careggi Hospital, University of Florence, Florence, Italy; ${ }^{16}$ Department of Gastroenterology, IRCCS, Casa Sollievo della Sofferenza Hospital, San Giovanni Rotondo, Italy; ${ }^{17}$ Upper Gastrointestinal Surgery, Humanitas Clinical and Research Hospital, IRCCS, Milan, Italy; ${ }^{18}$ San Raffaele Hospital, San Raffaele Vita-Salute University, Milan, Italy; ${ }^{19}$ Department of Gastroenterology, Istituto Clinico Humanitas, IRCCS, Milan, Italy; ${ }^{20}$ Division of Gastroenterology, Department of Clinical Medicine and Surgery, Federico II University Hospital School of Medicine, Naples, Italy; ${ }^{21}$ Department of Medicine B, University Hospital Münster, Münster, Germany; ${ }^{22}$ Department of Gastroenterology, DKD Helios Clinic Wiesbaden, Wiesbaden, Germany; ${ }^{23}$ Department of General-, Visceral-, Vascular and Pediatric Surgery, University of Würzburg, Würzburg, Germany; ${ }^{24}$ University of Groningen, University Medical Centre Groningen, Department of Genetics, Groningen, The Netherlands; ${ }^{25}$ Department of General, Visceral and Transplant Surgery, University Medical Center, University of Mainz, Mainz, Germany; ${ }^{26}$ Department of Medical Epidemiology and Biostatistics, Karolinska Institutet, Stockholm, Sweden; ${ }^{27}$ Department of Epidemiology, University Medical Center Utrecht, Utrecht, The Netherlands; ${ }^{28}$ Department of Medical Genetics, University Medical Center Utrecht, Utrecht, The Netherlands; ${ }^{29}$ Department of General and Abdominal Surgery, Protestant Hospital Castrop-Rauxel, Castrop-Rauxel, Germany; ${ }^{30}$ Institute for Medical Biometry, Informatics, and Epidemiology, University of Bonn, Bonn, Germany; ${ }^{31}$ Department of Clinical Sciences, Danderyds Hospital, Karolinska Institutet and Department of Surgery, Ersta Hospital, Stockholm, Sweden

32These authors contributed equally to this work.

${ }^{*}$ Correspondence: Dr J Schumacher, Institute of Human Genetics, University of Bonn, Sigmund-Freud-Str. 25, D-53127 Bonn, Germany, Tel: +49 228 28751028 , Fax: +49 228287 51011; E-mail: johannes.schumacher@uni-bonn.de

Received 16 March 2015; revised 26 October 2015; accepted 15 November 2015; published online 6 January 2016 
with a genetic susceptibility. ${ }^{1}$ Most recently, an insertion of eight amino acids in the cytoplasmic tail of HLA-DQ $\beta 1$ and two amino acid substitutions in the extracellular part of HLA-DQ $\alpha 1$ and HLA-DQ $\beta 1$ have been identified as being independently disease associated. ${ }^{2}$ Although on the cellular level the function of the identified achalasia risk variants remains speculative, the findings show that the HLA-DQ receptor and thereby autoimmune processes represent a major risk factor for idiopathic achalasia.

The aim of the present study was to assess whether the eight-aminoacid insertion in HLA-DQ $\beta 1$, which represents the strongest known achalasia risk variant, also confers risk in the Polish and Swedish population. For this purpose, we genotyped SNP rs28688207 (hg19 chr6:g.32628660T >C). The marker is located in a splice acceptor site and leads to an alternative splicing of HLA-DQB1 exon 5 (NM_001243961.1:c.773-1A>G), which encodes the risk-conferring eight-amino-acid insertion (details in Materials and Methods). The replication sample comprised 106 achalasia patients and 402 controls from Poland. In addition, we analyzed the insertion in 171 achalasia patients and 732 controls from Sweden. Beside the two cohorts from Poland and Sweden, we included samples from Central Europe (1042 patients and 3670 controls), Spain (273 patients and 327 controls) and Italy (165 patients and 1263 controls) that were part of our previous study, ${ }^{2}$ but have not been analyzed separately so far. Finally, we assessed the frequency and epidemiological consequences of the HLADQ $\beta 1$ insertion on the population level among the five different European cohorts.

\section{MATERIALS AND METHODS}

\section{Sample collection}

The case-control sample from Poland consisted of 106 patients and 402 controls. The Swedish cohort comprised 171 patients and 732 controls. In addition, samples from Central Europe (1042 patients and 3670 controls), Spain (273 patients and 327 controls) and Italy (165 patients and 1263 controls) were analyzed. The Central European sample consisted of 700 patients from Germany, 162 patients from Belgium and 180 patients from The Netherlands. All Central European controls were either from Germany $(N=2507)$ or from The Netherlands $(N=1163)$. The ethnic homogeneity of the Central European patients and controls has been previously shown. ${ }^{2}$ The distribution of females and males within each sample is presented in Supplementary Table 1. In all patients, achalasia diagnosis was made according to a standardized procedure, including esophageal manometry and/or esophagography.

\section{Genotyping and imputation}

Whereas the information of the HLA-DQ $\beta 1$ insertion (protein level) and rs28688207 (corresponding marker on the genetic level) has been obtained previously in the samples from Central Europe, Italy and Spain, ${ }^{2}$ the Polish and Swedish samples were analyzed for this variant within the present study. For this, we genotyped SNP rs28688207 using a Custom TaqMan Genotyping Assay
(Applied Biosystems) in the Polish case-control samples and the Swedish patient sample. The minor allele $G$ of $\mathrm{rs} 28688207$ (A/G, reverse strand) represents the achalasia risk allele whereby the $\mathrm{A}$ allele is the protective one. Allele A leads to an absence of $H L A-D Q B 1$ exon 5 (NM_001243961.1) and thereby to an absence of the risk-conferring eight amino acids on protein level (r.773_796del, p.(Pro259_Gly266del)), which is the case in the HLA-DQB1 transcript NM_002123.4. Of note, the given protein position 259-266 corresponds to position $227-234^{1}$ in the mature protein after cleavage the signal peptide with a size of 32 amino acids. To obtain the information of the insertion in the Swedish control cohort, we performed a HLA imputation with SNP2HLA (https://www.broadinstitute.org/mpg/snp2hla/). For this purpose, we used Illumina HumanHap 550 (San Diego, CA, USA) genotype data of 732 Sweden controls that have been collected for a genome-wide association study on breast cancer $\left(\right.$ CAHRES $=$ CAncer Hormone Replacement Epidemiology) ${ }^{3}$ For the imputation, a reference panel from the Type 1 Diabetes Genetics Consortium containing 5225 individuals of European descent was used. ${ }^{4}$ Previously, we have demonstrated that the imputation of the insertion show a high accuracy (concordance rate $=99.7 \%$ ). ${ }^{2}$ The data of the HLA-DQ $\beta 1$ insertion was deposited to the European Genome-phenome Archive (EGA, http://www.ebi.ac.uk/ega/) and is available under accession number EGAS00001001515.

\section{Biostatistics}

Armitage's trend test was used to compare genotype distributions between patients and controls in each population. The resulting $P$-values were combined by Fisher's combination test. The attributable risk (AR) was calculated as $\left(K_{\mathrm{p}} / f_{\mathrm{AA}}-1\right) /\left(K_{\mathrm{p}} / f_{\mathrm{AA}}\right) .{ }^{5}$ Whereas disease prevalence cannot be estimated from case-control studies, the ratio $K_{\mathrm{p}} / f_{\mathrm{AA}}$ is estimated by $\left(\left(\left(p_{\mathrm{I}} \times \mathrm{RR}\right)+1\right)-p_{\mathrm{I}}\right)^{2}$ with $p_{\mathrm{I}}$ denoting the population frequency of the insertion and RR denoting the allelic relative risk assuming a multiplicative mode of inheritance.

\section{RESULTS}

Analysis of rs28688207 in the Polish sample (106 patients and 402 controls) revealed a highly significant association with idiopathic achalasia $\left(P=1.84 \times 10^{-04}, \mathrm{RR}\right.$ (relative risk) $=3.52$, Table 1$)$. The frequency of the insertion was $7.1 \%$ in patients versus $2.1 \%$ in controls. Moreover, the insertion showed a strong association in the Swedish cohort (171 patients and 732 controls) with $P=7.44 \times 10^{-05}$ $(\mathrm{RR}=2.93$, Table 1). In Sweden, the insertion occurred at a frequency of $6.1 \%$ in patients and only $2.2 \%$ in controls.

Combining all five European data sets - in total 1757 patients and 6394 controls from Central Europe, Italy, Spain, Poland and Sweden the insertion was strongly associated with achalasia $\left(P_{\text {combined }}=1.67\right.$ $\left.\times 10^{-35}\right)$. However, the frequency of the insertion was substantially lower in the northern compared with southern European populations and thereby showed a geospatial north-south gradient (Figure 1). In comparison to Sweden, the insertion was nearly four times more common in the Italian controls $(8 \%)$ and more than twice as high in patients from Italy $(16.1 \%)$. The insertion is achalasia associated in

Table 1 The results of the eight-amino-acid insertion in HLA-DQ $\beta 1$ are given for the five screened European populations. For patients and controls separately, the total number of studied samples $(N)$, the distribution of the insertion as follows $A A / P P / P P(A=$ insertion absent, $\mathbf{P}=$ insertion present) and the frequency of the insertion are shown

\begin{tabular}{|c|c|c|c|c|c|c|c|c|}
\hline \multirow[b]{2}{*}{ Population } & \multicolumn{2}{|c|}{ Patients } & \multicolumn{2}{|c|}{ Controls } & \multirow[b]{2}{*}{$\mathrm{P}$} & \multirow[b]{2}{*}{$R R$} & \multirow[b]{2}{*}{$A R(\%)$} & \multirow[b]{2}{*}{$\mathrm{K}_{p} / \mathrm{f}_{A A}$} \\
\hline & N (distribution) & Frequency (\%) & N (distribution) & Frequency (\%) & & & & \\
\hline Italy & $165(115 / 47 / 3)$ & 16.1 & $1263(1069 / 187 / 7)$ & 8.0 & $1.00 \times 10^{-06}$ & 2.21 & 16.8 & 1.202 \\
\hline Spain & $273(223 / 45 / 5)$ & 10.1 & $327(294 / 33 / 0)$ & 5.0 & $1.13 \times 10^{-03}$ & 2.11 & 10.3 & 1.115 \\
\hline Central Europe & $1042(873 / 157 / 12)$ & 8.7 & $3670(3432 / 235 / 3)$ & 3.3 & $3.03 \times 10^{-25}$ & 2.80 & 10.9 & 1.122 \\
\hline Poland & $106(91 / 15 / 0)$ & 7.1 & $402(385 / 17 / 0)$ & 2.1 & $1.84 \times 10^{-04}$ & 3.52 & 9.9 & 1.109 \\
\hline Sweden & $171(150 / 21 / 0)$ & 6.1 & $732(700 / 32 / 0)$ & 2.2 & $7.44 \times 10^{-05}$ & 2.93 & 7.9 & 1.086 \\
\hline
\end{tabular}

In addition, the $P$-values of the association test $(P)$, the effect sizes as relative risk (RR), the attributable risks (AR) and the ratio $K_{\mathrm{p}} / f_{\mathrm{AA}}$ between disease prevalence $K_{\mathrm{D}}$ and disease penetrance $f_{\mathrm{AA}}$ for non-carriers of the insertion are given. 


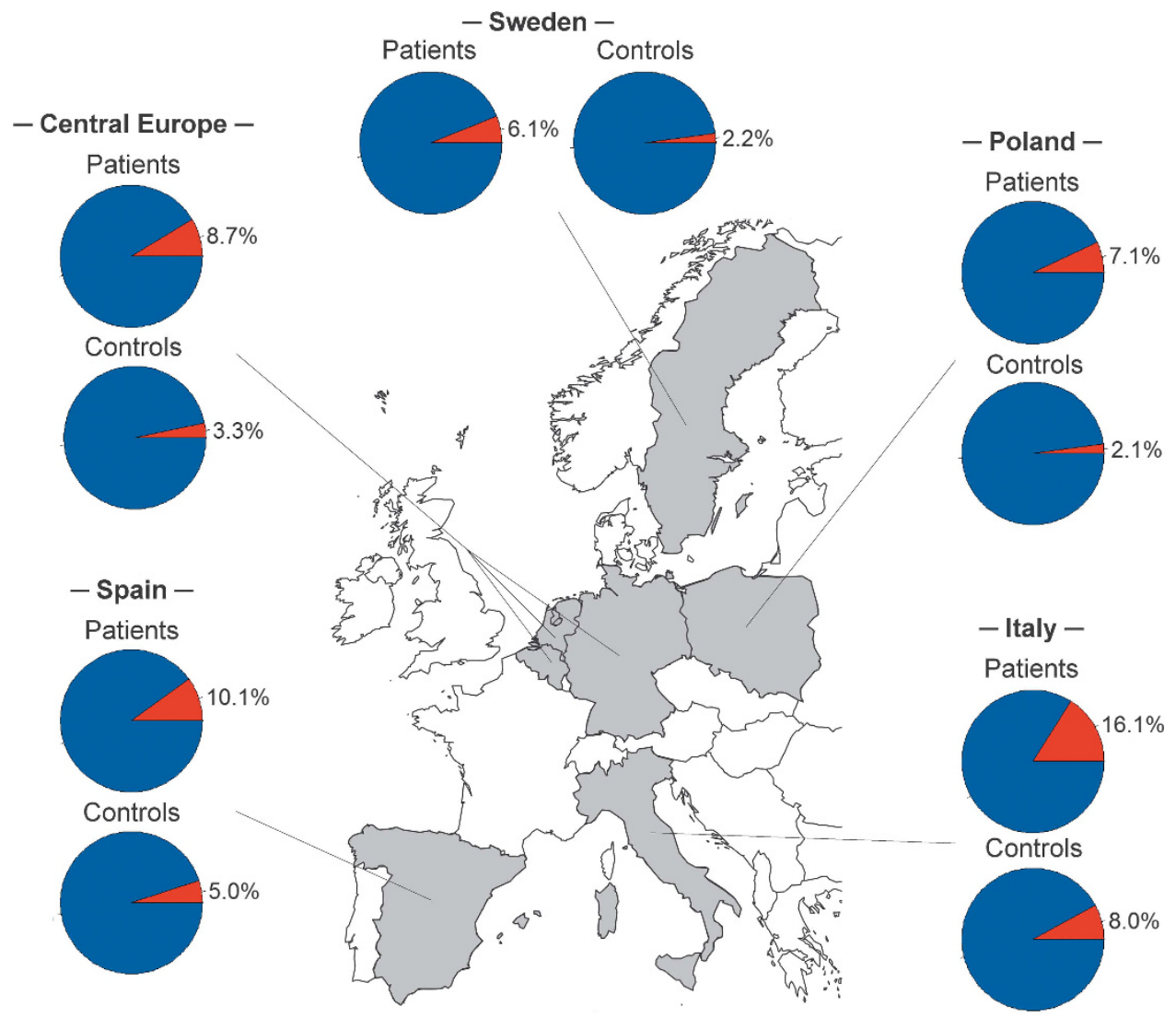

Figure 1 The frequencies of the eight-amino-acid HLA-DQ 1 insertion are given for the five screened European populations. In every population, the insertion is more frequent in achalasia patients compared with controls and is strong disease associated (Table 1). However, the frequency differs among European populations with a geospatial north-south gradient. The lowest frequency was observed for the Swedish and Polish samples $(\sim 6-7 \%$ in patients and $\sim 2 \%$ in controls). In contrast, the frequency was considerably higher in Italy ( 16\% in patients and $8 \%$ in controls).

the Italian population with $P=1.00 \times 10^{-06}(\mathrm{RR}=2.21$, Table 1$)$. In Spain, the frequency of the insertion was $5.0 \%$ and $10.1 \%$ in controls and patients, respectively, which is around two times higher compared with northern Europeans. In the Spanish population the insertion showed achalasia association with $P=1.13 \times 10^{-03}$ $(\mathrm{RR}=2.11$, Table 1$)$. In contrast, the insertion showed a similar frequency in controls $(3.3 \%)$ and in patients $(8.7 \%)$ from Central Europe compared with Poland, which is approximately on the same latitude. The insertion is achalasia associated in Central Europe with $P=3.03 \times 10^{-25} \quad(\mathrm{RR}=2.80$, Table 1$)$. Apart from the observed geospatial frequency differences, the effect sizes of the insertion did not differ substantially between populations $(\mathrm{RR}=2.11-3.52$, Table 1$)$. Next, we asked whether the differences in the insertion frequencies affect the prevalence of achalasia among the five populations. We observed that the AR, which defines the proportion of patients that would be not affected in the absence of the insertion, differs between the most southern Italian and the most northern Swedish population. In the Italian population the $\mathrm{AR}$ is $16.8 \%$, whereas the $\mathrm{AR}$ is $7.9 \%$ in Sweden (Table 1). Considering the insertion only, we estimate that $\sim 10.7 \%$ more patients with idiopathic achalasia may exist in Italy compared with Sweden.

\section{DISCUSSION}

We show that the eight-amino-acid insertion in HLA-DQ $\beta 1$ also confers achalasia risk in the Polish and Swedish population. In addition, we observe that the insertion's frequency shows a geospatial north-south gradient in Europe. The geospatial north-south gradient implies that the prevalence of idiopathic achalasia may differ among populations. Using data of the 1000 Genomes Project, we compared our findings with frequency data of the 26 different samples which belong to the five super-populations Africa, America, Asia, South Asia and Europe. This resulted in a coherent picture for Europe, where the frequency of the insertion is lowest in the northern (ie, $1 \%$ in Finland) and highest in the southern European population (ie, 6\% in Italy, Supplementary Figure 1). Latitudinal frequency gradients of genetic risk variants have been previously found for other autoimmune diseases, where genetic variation at the HLA locus has an important role in disease pathology. For example, an opposite gradient has been observed for type-1 diabetes mellitus and IgA nephropathy, which are more common in northern compared with southern European populations. ${ }^{6,7}$ As these disorders also show differences in disease prevalence among world populations, it is interesting to note that the HLA-DQ $\beta 1$ insertion shows the highest frequency in the South Asian populations (Supplementary Figure 1) suggesting a possible higher prevalence rate of achalasia. However, to our knowledge, a higher prevalence/incidence rate in the Asian population compared with the European population has not been reported so far. In total, three studies have focused on incidence/prevalence rates in the Asian population, whereby two of them studied a comparably small cohort $\left(N<50\right.$ achalasia patients). ${ }^{8,9}$ The other study from Kim and colleagues $^{10}$ was based on an achalasia cohort of 3105 patient diagnosed between 2007 and 2011 in Korea. The authors estimated a prevalence of 7.8/100 000 which is comparable to the rates observed in Europe. However, future studies on large sample sizes - especially 
in South Asia - have to prove whether there are differences between the achalasia prevalence in Asia and other populations. Furthermore, it still has to be shown that the insertion also confers achalasia risk in the Asian population and thereby may affect the disease prevalence. Evolutionary mechanisms such as genetic drift or natural selection in the context of different environmental conditions are plausible explanations for frequency differences of genetic variants between populations.

In conclusion, we show that the strongest risk variant for idiopathic achalasia identified so far, the eight-amino-acid insertion in the cytoplasmic tail of HLA-DQ $\beta 1$, also confers disease risk in the Polish and Swedish population. With this study, we replicate our initial finding in two independent samples. In addition, we found that the frequency of the insertion substantially differs among European populations with a geospatial north-south gradient. Although our study provides further insights into the genetic disease architecture, the data implicate that much more work is necessary to elucidate the etiological complexity of idiopathic achalasia.

\section{CONFLICT OF INTEREST}

The authors declare no conflict of interest.

\section{ACKNOWLEDGEMENTS}

We thank all patients for participating in this study. We acknowledge our collaborating clinical partners, all colleagues who contributed to the patient's recruitment, our laboratory technicians and colleagues responsible for database management. We thank the Type I Diabetes Genetics Consortium (T1DGC) for data access. IG, MK and JS received support for this work from the Deutsche Forschungsgemeinschaft (DFG), individual grants GO 1795/1-1, KN 378/2-1 and SCHU 1596/5-1. MMN received support for this work from the
Alfried Krupp von Bohlen und Halbach-Stiftung, and is a member of the DFG funded Excellence Cluster ImmunoSensation. PIWdB is the recipient of a Vernieuwingsimpuls VIDI Award from the Netherlands Organization for Scientific Research (NWO). MMW is supported by a postdoctoral fellowship of the Fund for Scientific Research (FWO) Flanders, Belgium. GEB is supported by a grant from the Research Foundation - Flanders (FWO, Odysseus program). AT is supported by grants from the Erling-Persson Family Foundation, Sweden. The Heinz Nixdorf Recall cohort was established with the generous support of the Heinz Nixdorf Foundation, Germany.

1 Gockel HR, Schumacher J, Gockel I, Lang H, Haaf T, Nothen MM: Achalasia: will genetic studies provide insights? Hum Genet 2010; 128: 353-364.

2 Gockel I, Becker J, Wouters MM et al: Common variants in the HLA-DQ region confer susceptibility to idiopathic achalasia. Nat Genet 2014; 46: 901-904.

3 Einarsdottir K, Humphreys K, Bonnard $\mathrm{C}$ et al: Linkage disequilibrium mapping of CHEK2: common variation and breast cancer risk. PLoS Med 2006; 3: e168.

$4 \mathrm{Jia}$ X, Han B, Onengut-Gumuscu S et al: Imputing amino acid polymorphisms in human leukocyte antigens. PLoS One 2013; 8: e64683.

5 Schaid DJ, Sommer SS: Genotype relative risks: methods for design and analysis of candidate-gene association studies. Am J Hum Genet 1993; 53: 1114-1126.

6 Borchers AT, Uibo R, Gershwin ME: The geoepidemiology of type 1 diabetes. Autoimmun Rev 2010; 9: A355-365.

7 Kiryluk K, Li Y, Sanna-Cherchi S et al: Geographic differences in genetic susceptibility to IgA nephropathy: GWAS replication study and geospatial risk analysis. PLoS Genet 2012; 8: e1002765.

8 Ho KY, Tay HH, Kang JY: A prospective study of the clinical features, manometric findings, incidence and prevalence of achalasia in Singapore. J Gastroenterol Hepatol 1999; 14: 791-795.

9 Farrukh A, DeCaestecker J, Mayberry JF: An epidemiological study of achalasia among the South Asian population of Leicester, 1986-2005. Dysphagia 2008; 23: $161-164$.

10 Kwon HY, Lim JH, Shin YW, Kim CW: A case of chronic cough caused by achalasia misconceived as gastroesophageal reflux disease. Allergy Asthma Immunol Res 2014; 6: 573-576.

Supplementary Information accompanies this paper on European Journal of Human Genetics website (http://www.nature.com/ejhg) 